Asian J. Med. Biol. Res. 2021, 7 (3), 230-237; doi: 10.3329/ajmbr.v7i3.56133

\author{
Asian Journal of \\ Medical and Biological Research \\ ISSN 2411-4472 (Print) 2412-5571 (Online) \\ www.ebupress.com/journal/ajmbr
}

Article

\title{
Association of the dominant handgrip strength with the hand dimensions of adult Bangladeshi female laborers
}

\author{
Mst. Taslima Akter ${ }^{1 *}$, Nahid Farhana Amin ${ }^{2}$ and Ahsan Arif ${ }^{3}$ \\ ${ }^{\mathbf{1}}$ Assistant Professor, Department of Anatomy, TMSS Medical College, Bogura, Bangladesh \\ ${ }^{2}$ Professor, Department of Anatomy, Bangabandhu Sheikh Mujib Medical University (BSMMU), Dhaka, \\ Bangladesh \\ ${ }^{3}$ Assistant Register, Department of Surgery, Shaheed Ziaur Rahman Medical College, Bogura, Bangladesh \\ "Corresponding author: Dr. Mst. Taslima Akter, Assistant Professor, Department of Anatomy, TMSS Medical \\ College, Bogura, Bangladesh. Phone: +8801774046369; E-mail: dr.taslima1808@ gmail.com
}

Received: 14 July 2021/Accepted: 03 August 2021/ Published: 30 September 2021

\begin{abstract}
The handgrip strength determines the overall physical health and muscle function of the hand. Nowadays in Bangladesh, female laborers are an important source of work force in many industries. Laborers require more handgrip strength to perform their daily work efficiently. Besides, hand is the part of the body that directly related with handgrip strength, so, the hand dimensions are deemed essential to investigate. Therefore this study is to investigate the association of the dominant handgrip strength with the hand dimensions like hand length, hand breadth, hand span, palm length, palm breadth and wrist breadth of adult Bangladeshi female laborers. Hundred (100) adult female laborers aged between 18 to 45 years, residing in different slums in Dhaka city of Bangladesh were the participants of the present research. Six selected hand dimensions of the right side were measured using the digital slide caliper by direct physical procedure and dominant handgrip strength was measured using a digital handgrip dynamometer. The associations of the dominant handgrip strength with the selected six hand dimensions were tested using Pearson's correlation coefficient test. The mean value of the dominant handgrip strength of the laborers was $25.6 \mathrm{~kg}$. In present research, the dominant handgrip strength was positively associated with the six selected hand dimensions (hand length, hand breadth, hand span, palm length, palm breadth and wrist breadth). However, the association of dominant handgrip strength with the breadth of hand, palm and wrist reached up to significant level individually. Besides, to get desired success in work of a female laborer, it is important to see the hand anthropometry and handgrip strength, because, better handgrip strength lead to better performance in work.
\end{abstract}

Keywords: handgrip strength; hand dimensions; female laborer

\section{Introduction}

In human body, the hand is one of the complex and beautiful pieces of natural engineering (McGavin, 2014). It has the capability of wide variety of functions, including gross and fine motor functions and also has the capability of relaying sensory information to the brain about the temperature, the shape and texture of objects (Barut et al., 2008). However, fine motor movements enable us to perform delicate tasks, such as holding small objects or performing detailed work. Besides, the fingers of hand help to judge how firmly to hold anything. Only human can bring the thumbs across the hand to connect with other fingers to make a forceful handgrip (Almecija and Sherwood, 2018). Therefore, the handgrip is the result of forceful flexion of all finger joints with the maximum voluntary force that the participant can exert under normal biokinetic conditions, which uses several muscles in hand and forearm (Koley and Kaur, 2010). Handgrip strength is an important indicator of the overall body strength of an individual (Neves et al., 2017). Moreover, hands are important tools for performing various works. Its morphological and functional properties play an important role in performance. For a developing country like Bangladesh, female laborer is a great source of working manpower of Bangladesh in 
many industries, construction works, field works and even in household works (Islam, 2018). On the other hand, the size of the female labor force in Bangladesh has increased at a significantly higher rate than that of the male from 2015-16 (Islam, 2018). Laborers require more handgrip strength to perform their daily work efficiently. Therefore, without adequate handgrip and forearm strength, the works do not meet its desired success. Human hands are the part of the body that directly related with the handgrip strength. So, the investigation of the association between hand dimensions and dominant handgrip strength is essential. In clinical practice, handgrip strength is a screening tool for women at risk of osteoporosis, musculoskeletal deformities, and neuromuscular disorders, evaluation of pre- and post-operative patients (Fallahi and Jadidian, 2011). Besides, handgrip strength is used in the investigation and follow-up of a patient with musculoskeletal and neuromuscular disease of the upper limb and also important in determining the efficacy of different treatment strategies of hand and hand rehabilitation (Oseloka et al., 2014). Moreover, in musculoskeletal, nervous, or joint disorders the handgrip strength test is a simple and economical test that gives practical information (Bansode et al., 2014). However, normative values of the dominant handgrip strength of the western countries and different ethnic groups might not be applicable for Bangladeshis because of differences in socio-cultural aspects. Therefore, such type of research was planned to provide a baseline quantitative data on the dominant handgrip strength as well as to find out the association of dominant handgrip strength and some selected hand dimensions in Bangladeshi female laborers.

\section{Materials and Methods}

\subsection{Research design}

A cross-sectional study was carried out in 100 adults Bangladeshi female laborers aged between 18 to 45 years, residing different slum areas at Mirpur in Dhaka city during March 2019 to February 2020. All measurements were taken during a particular time ( $3 \mathrm{pm}$ to $6 \mathrm{pm}$ ) of the day to prevent diurnal variations. Data analysis was carried out in the Department of Anatomy, Bangabandhu Sheikh Mujib Medical University (BSMMU), Dhaka. In the study, right-handed participant was selected. Participants with the history and physical features of any congenital anomaly, genetic disorder, neurological disorder, endocrine disorder, autoimmune disorder, trauma, surgery, musculoskeletal impairment that can affect the hand anthropometry was excluded from the current research. The ethical clearance was obtained from Institutional Review Board of BSMMU.

\subsection{Anthropometric measurements}

To measure hand dimensions, the participants was asked to place the hand on a table and the dimensions were measured with a digital sliding caliper. The length of the hand was measured as the linear projected distance from the mid-point of the distal wrist crease to the most anterior projecting point on the middle finger. The breadth of the hand was measured as the linear projected distance of the hand between the most lateral point on the head of second metacarpal bone and the most medial point of the head of fifth metacarpal bone. The hand span was measured as linear distance from the tip of the thumb to the tip of the little finger, when the hand was placed on a flat surface and fingers were abducted and extended as far as possible. The palm length was measured from the midpoint of the distal wrist crease to the midpoint of the proximal or basal crease of the middle finger. The palm breadth was measured as a linear distance extending from the lateral end of the proximal transverse crease of the palm or thinner crease that cuts the radial border of the hand to the medial end of the distal transverse crease of the palm that cuts the ulnar border of the hand. The wrist breadth was measured as the distance between the ulnar and radial styloid processes of the hand. The handgrip strength of dominant hand was measured using a standard adjustable digital handgrip dynamometer (Camry, Model no. EH 101). Before taking the measurement, the participant was asked to stand comfortably with the shoulder adducted and neutrally rotated and elbow in full extension. After taking a deep breath, the participant was requested to hold the handgrip dynamometer freely in dominant hand without any support, and not touching her trunk (Figure 1). Then, the participant was asked to squeeze the handle of dynamometer with maximum strength as hard as possible without moving the body and hold it for 4- 5 seconds. The instrument automatically records the highest measurement in kilogram during the whole procedure and displays that in monitor. After 30 seconds break the participant was again requested to repeat the procedure.

\subsection{Statistical analysis}

The statistical analysis was performed using SPSS software (version 24.0) and the association of the dominant handgrip strength with the selected six hand dimensions were tested using Pearson's correlation coefficient test. 


\section{Results and Discussion}

In Table 1, the mean values as well as ranges of the hand dimensions and dominant handgrip strength of Bangladeshi female laborers were described. The mean length, breadth and span of the hand were $16.47 \pm 0.57$ $\mathrm{cm}, 7.11 \pm 0.34 \mathrm{~cm}$ and $17.35 \pm 0.76 \mathrm{~cm}$ respectively. The mean breadth of the wrist was $4.80 \pm 0.26 \mathrm{~cm}$. Again, the mean length and breadth of the palm were $9.42 \pm 0.39 \mathrm{~cm}$ and $7.54 \pm 0.33 \mathrm{~cm}$ respectively. The mean dominant handgrip strength was $25.59 \pm 3.24 \mathrm{~kg}$.

The correlation coefficient (r) and $\mathrm{p}$ - value of the selected hand dimensions shown in Table 2. Correlations of the dominant handgrip strength (DHGS) with each of the selected hand dimensions were positive but the correlation of dominant handgrip strength with the breadth of hand $(r=0.373 ; p=0.000)$, palm $(r=0.194 ; p=$ $0.000)$ and wrist $(r=0.562 ; p=0.000)$ reached up to significant level individually. However, the correlation of dominant handgrip strength with the length $(\mathrm{r}=0.168 ; \mathrm{p}=0.094)$, span of hand $(\mathrm{r}=0.145 ; \mathrm{p}=0.150)$ and length of palm $(r=0.194 ; p=0.053)$ were weak.

The correlations of the dominant handgrip strength with each of the selected hand dimensions of Bangladeshi female laborers were assessed and displayed using scatter diagrams in Figure 2 to Figure 7. In present research, the dominant handgrip strength had non-significant positive correlation with the length of the hand $(\mathrm{r}=+0.168$, $\left.\mathrm{r}^{2}=0.028, \mathrm{p}=0.094\right)$; with the hand span

$\left(r=+0.145, r^{2}=0.021, p=0.150\right)$ and with the length of the palm $\left(r=+0.194, r^{2}=0.038, p=0.053\right)$ individually. But the dominant handgrip strength had significant positive correlation with breadth of the hand $(\mathrm{r}$ $\left.=+0.373, r^{2}=0.139, p=0.000\right)$, with breadth of wrist $\left(r=+0.562, r^{2}=0.316, p=0.000\right)$ and with breadth of palm $\left(\mathrm{r}=+0.423, \mathrm{r}^{2}=0.179, \mathrm{p}=0.000\right)$ individually.

In the present research, in female laborers, there was a positive significant association was found between the dominant handgrip strength and the hand breadth. But, the correlation of the dominant handgrip strength with the length of the hand and hand span were positive individually but non-significant. Similarly Rawat et al. (2016) found a significant correlation was between the handgrip strength and the hand breadth rather than the hand length of 375 Indian office going females. The finding of the present research also consistent with several researches (Vivekanand et al., 2016; Sandhu et al., 2017). In contrast, Shah et al. (2012) observed that there is a significant association between the handgrip strength with all hand dimensions (hand length and span) except hand breadth. Koley and Singh (2009) established that the dominant handgrip strength significantly correlated with hand length and breadth in 18-25 years aged healthy Indian collegiate females. The contradictory finding of Shah et al. (2012) and Koley and Singh (2009) with the present research was unknown.

In case of hand span, Bansode et al. (2014) agreed that the dominant handgrip strength had a significant association with the hand span on 121 Indian healthy adult populations. Ruiz et al. (2006) showed that hand span influences the handgrip strength. Those finding were similar to present research. After repeated workload the muscle mass is increased, which lead to increase handgrip strength. Thus, a muscle with a large crosssectional area is able to produce maximal force during working (Rawat et al., 2016). A large hand span provides a large surface area during grasping an object in a grip associated work. In the present research, female laborers often do much work which require the more grip strength.

In female laborers, a positive significant correlation was found between the dominant handgrip strength and the wrist breadth. The finding of the present research was in accordance with Shah et al. (2012) conducted a research on 50 Indian females, where there was a significant correlation between the dominant handgrip strength and wrist breadth. To generate adequate strength, functional efficacy of wrist breadth is important. According to the present research, increased wrist breadth was directly related to increased handgrip strength of female laborers.

In this research, a positive significant correlation was found between the dominant handgrip strength and the palm breadth in the female laborers. But there was a positive non-significant correlation was observed between the dominant handgrip strength and palm length of the participants. The finding of present research was consistent with the findings of 40 male athletes and 40 non-athletes in Iran, observed by Fallahi and Jadidian (2011). As, after repeated workload the muscle mass is increased, which lead to increase handgrip strength (Aadahl et al., 2011). Besides, a muscle with a large cross-sectional area is able to produce maximal force during working. Moreover, the muscle of the palm is 'pennate' in shape, which allows producing more force during griping activity. Because of more use of palm by the female laborers, the muscles of palm become hypertrophied, thus cause increased in palm breadth. In addition, in the present research, each participant of female laborers had the history of doing their selected work since at least one year. 
Table 1. Values of the hand dimensions and dominant handgrip strength in Bangladeshi female laborers $(\mathbf{n}=\mathbf{1 0 0})$.

\begin{tabular}{lll}
\hline Variable & Range & Mean $( \pm \text { SD })^{*}$ \\
\hline Length of the hand $(\mathrm{cm})$ & $15.05-18.55$ & $16.47( \pm 0.57)$ \\
Breadth of the hand $(\mathrm{cm})$ & $6.10-7.76$ & $7.11( \pm 0.34)$ \\
Hand span $(\mathrm{cm})$ & $15.94-20.60$ & $17.35( \pm 0.76)$ \\
Breadth of the wrist $(\mathrm{cm})$ & $4.15-5.85$ & $4.80( \pm 0.26)$ \\
Length of the palm $(\mathrm{cm})$ & $8.64-10.42$ & $9.42( \pm 0.39)$ \\
Breadth of the palm $(\mathrm{cm})$ & $6.70-8.16$ & $7.54( \pm 0.33)$ \\
Dominant handgrip strength $(\mathrm{kg})$ & $18.03-37.8$ & $25.59( \pm 3.24)$ \\
\hline
\end{tabular}

SD*: Standard Deviation

Table 2. Correlation coefficient of the dominant handgrip strength (DHGS) with the hand dimensions of Bangladeshi female laborers $(n=100)$.

\begin{tabular}{lll}
\hline Variable & Correlation coefficient $(\mathbf{r})$ & $\begin{array}{l}\text { Significance (p value) of } \\
\text { correlation with DHGS }\end{array}$ \\
\hline Length of the hand $(\mathrm{cm})$ & 0.168 & 0.094 \\
Breadth of the hand $(\mathrm{cm})$ & 0.373 & 0.000 \\
Hand span $(\mathrm{cm})$ & 0.145 & 0.150 \\
Breadth of the wrist $(\mathrm{cm})$ & 0.562 & 0.000 \\
Length of the palm $(\mathrm{cm})$ & 0.194 & 0.053 \\
Breadth of the palm $(\mathrm{cm})$ & 0.423 & 0.000 \\
\hline
\end{tabular}

If $\mathrm{p} \leq 0.05$, it is considered as significant.

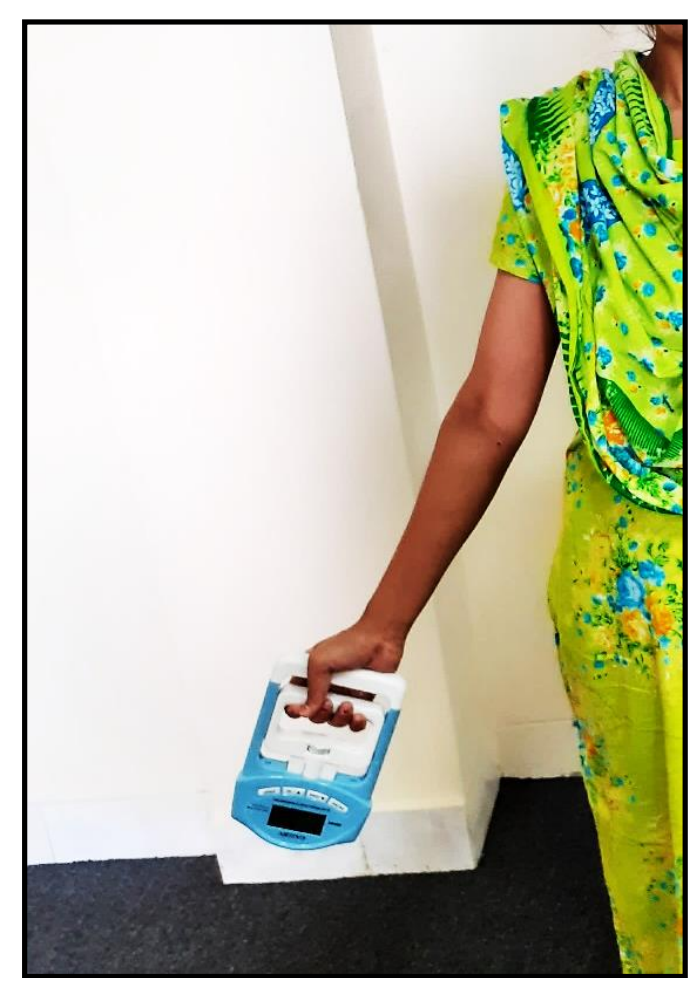

Figure 1. Procedure of measuring the dominant handgrip strength using digital handgrip dynamometer. 


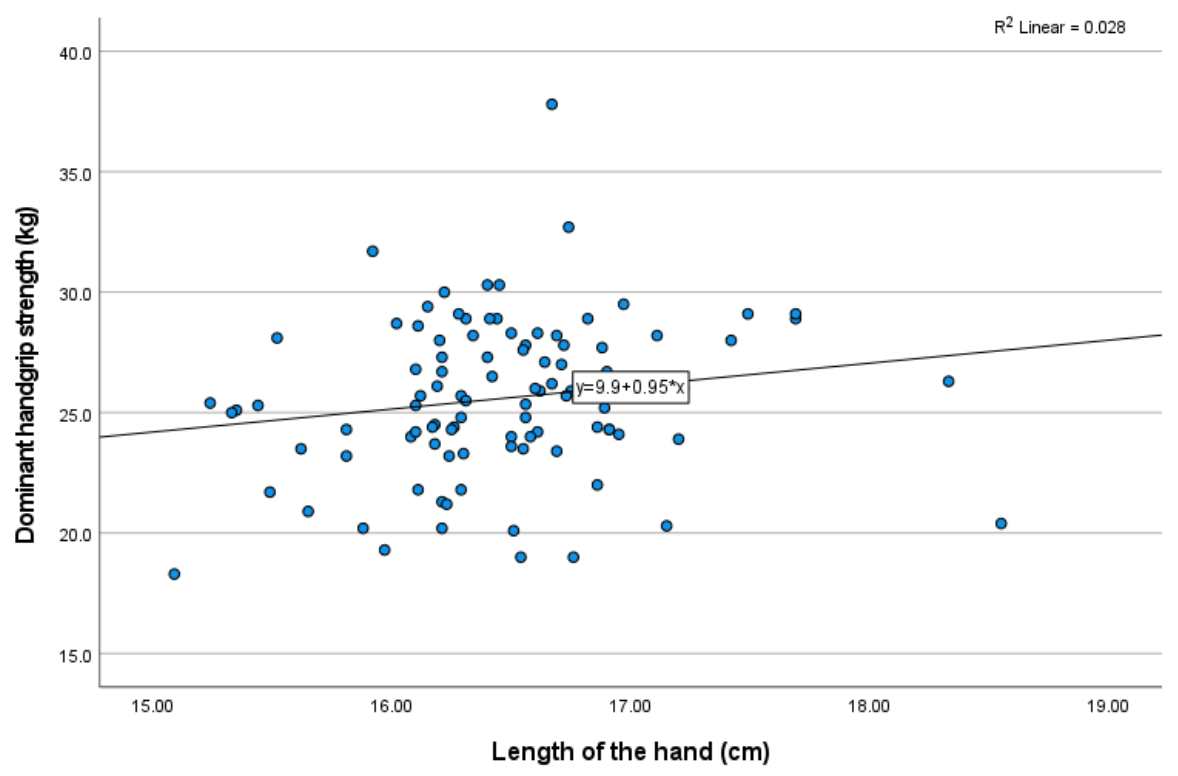

Figure 2. Non-significant positive correlation $\left(r=+0.168, r^{2}=0.028, p=0.094\right)$ of the dominant handgrip strength with the length of the hand of Bangladeshi female laborers.

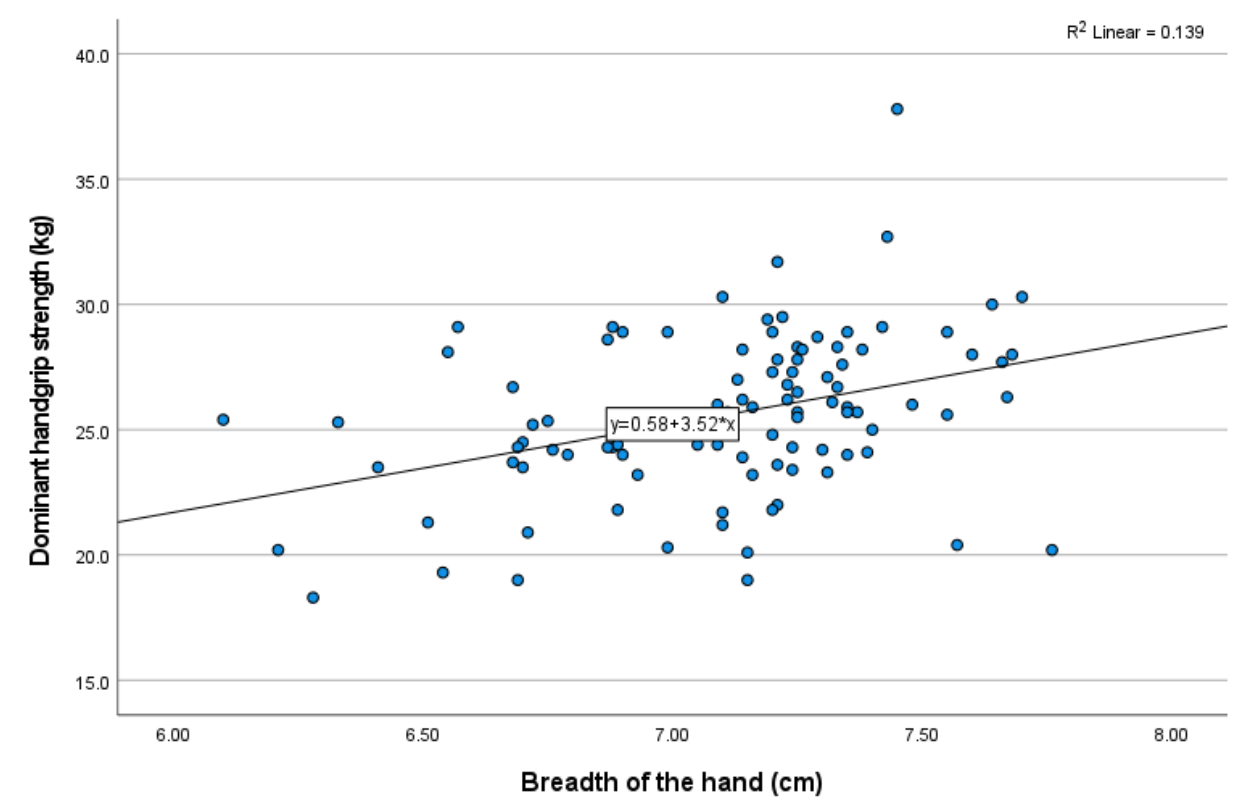

Figure 3. Significant positive correlation $\left(r=+0.373, r^{2}=0.139, p=0.000\right)$ of the dominant handgrip strength with breadth of the hand of the Bangladeshi female laborers. 


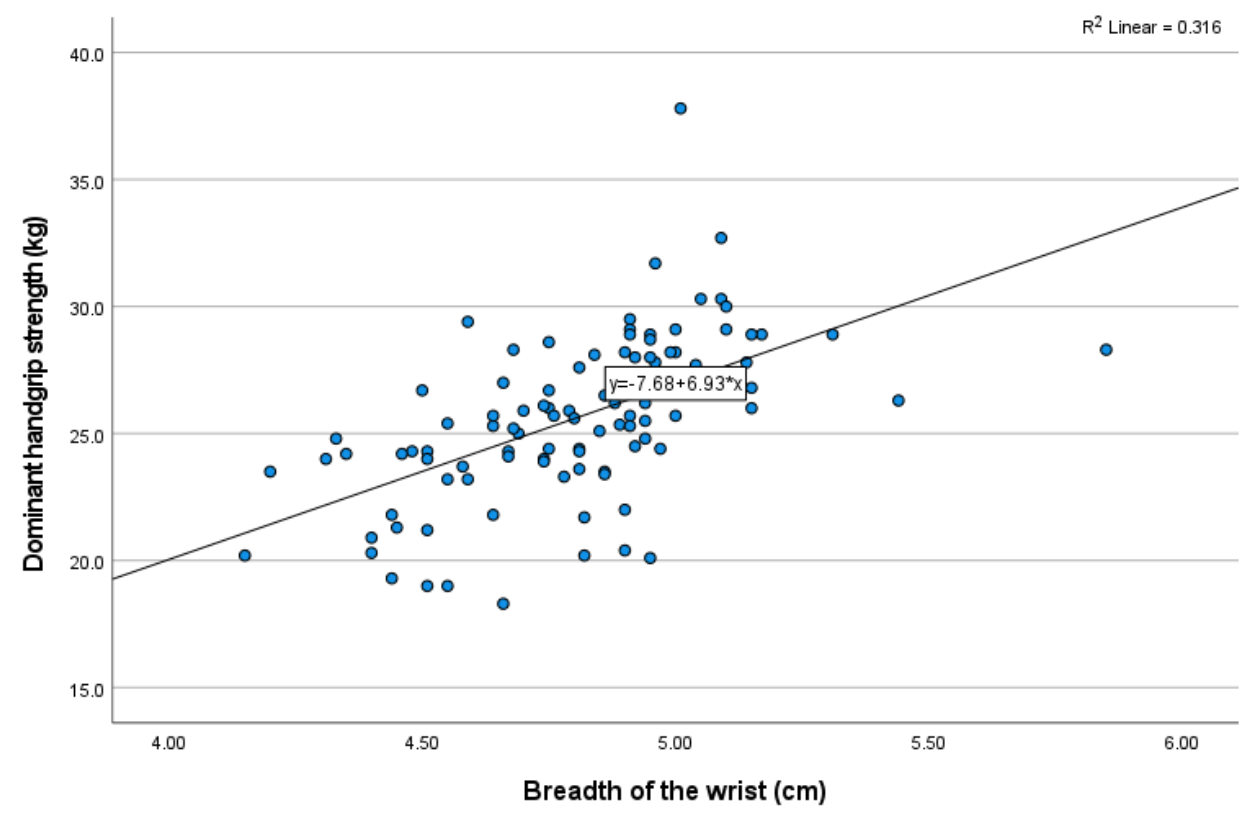

Figure 4. Significant positive correlation $\left(r=+0.562, r^{2}=0.316, p=0.000\right)$ of the dominant handgrip strength with breadth of the wrist of the Bangladeshi female laborers.

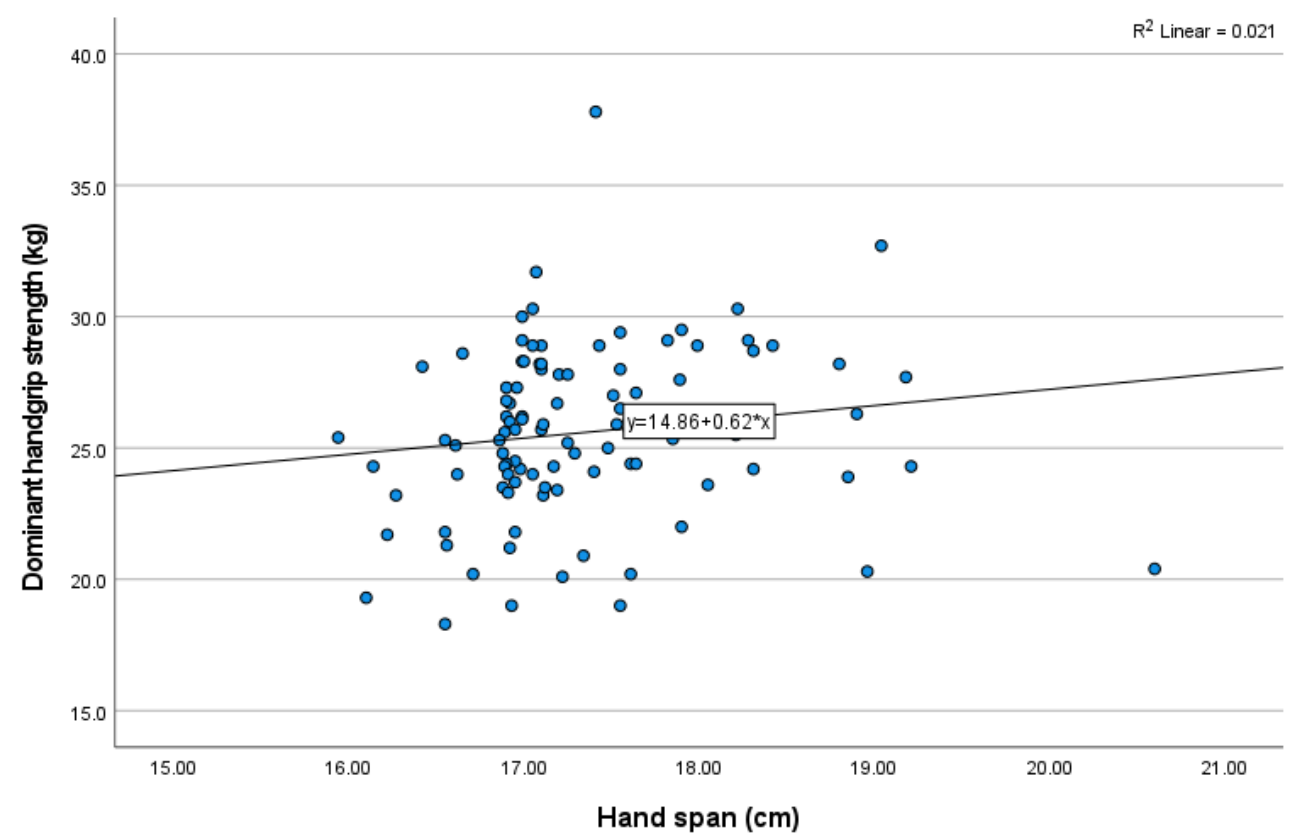

Figure 5. Non-significant positive correlation $\left(r=+0.145, r^{2}=0.021, p=0.150\right)$ of the dominant handgrip strength with hand span of the Bangladeshi female laborers. 


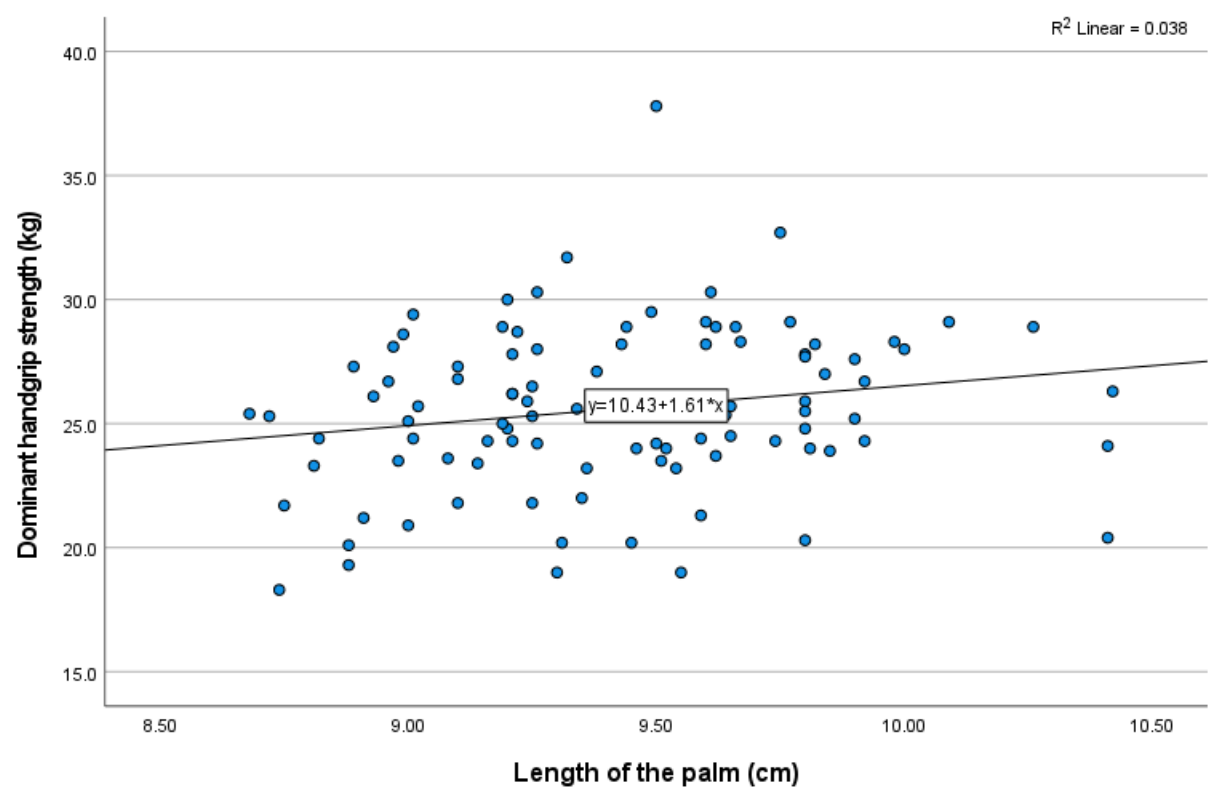

Figure 6. Non-significant positive correlation $\left(r=+0.194, r^{2}=0.038, p=0.053\right)$ of the dominant handgrip strength with length of the palm of the Bangladeshi female laborers.

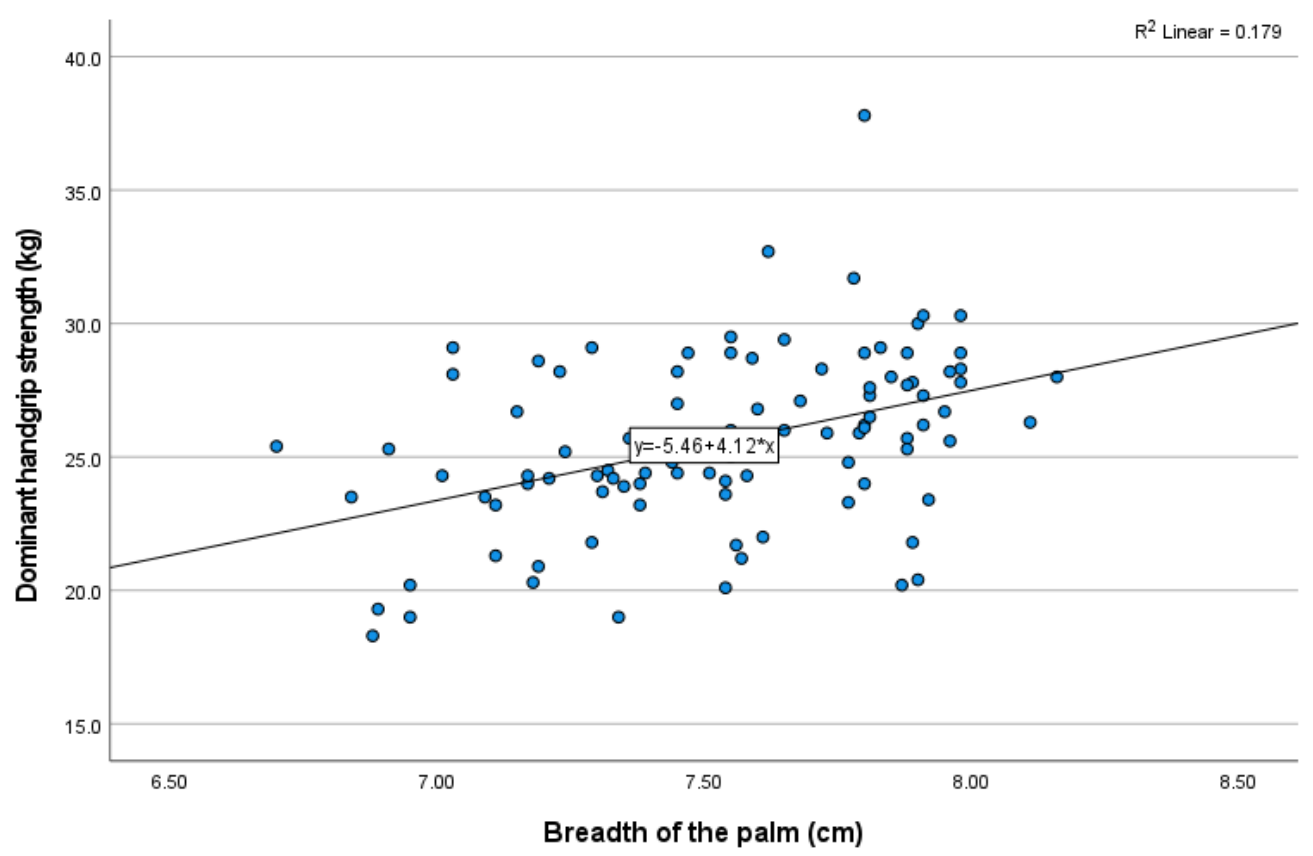

Figure 7. Significant positive correlation $\left(r=+0.423, r^{2}=0.179, p=0.000\right)$ of the dominant handgrip strength with breadth of the palm of the Bangladeshi female laborers.

\section{Conclusions}

In present research, the dominant handgrip strength was positively associated with the six selected hand dimensions (hand length, hand breadth, hand span, palm length, palm breadth and wrist breadth). However, the association of dominant handgrip strength with the breadth of hand, palm and wrist reached up to significant level individually. Furthermore, to get desired success in work of a female laborer, it is important to see the body measurements and handgrip strength. Because, better handgrip strength lead to better performance in work. The results of the present research can provide a baseline data for a normative representation regarding selected hand dimensions and dominant handgrip strength of Bangladeshi female laborers, which is necessary in 
clinical practice of neurological, musculoskeletal disease and upper limb rehabilitation. It is also helpful for physiotherapist, nutritionist and possible screening of other health issues. Besides, it may help in developing different kinds of hand machine and tools for females. These should encourage others in taking up further research in this field.

\section{Acknowledgements}

This work was supported by a grand in aid from Bangabandhu Sheikh Mujib Medical University (BSMMU), Dhaka, Bangladesh. The authors also thanks to the women participants of this research for their time and effort that enable the completion of this research.

\section{Conflict of interest}

None to declare.

\section{Authors' contribution}

Mst. Taslima Akter designed the study, sample collection, interpretation of data, and drafting the write up of the article. Nahid Farhana Amin guided and directed in different steps of the study. Ahsan Arif helped in data collection and statistical procedure for this research. All authors read and approved the final manuscript.

\section{References}

Aadahl M, Beyer N, Linneberg A, Thuesen BH and Jorgensen T, 2011. Grip strength and lower limb extension power in 19- 72-year-old Danish men and women: the Health 2006 study. BMJ Open, 2: 1-6.

Almecija S and CC Sherwood, 2018. Hands, brains, and precision grips: Origins of tool use. Behaviors, 3: 299313.

Bansode DG, LJ Borse and RD Yadav, 2014. Study of correlation between dominant hand's grip strength and some physical factors in adults' males and females. Int. J. Pharma. Res. Heal. Sci., 2: 316-323.

Barut C, P Demirel and S Kiran, 2008. Evaluation of hand anthropometric measurements and grip strength in basketball, volleyball, and handball players. Int. J. Experi. And Clin. Ana., 2: 55-59.

Fallahi AA and AA Jadidian, 2011. The effect of hand dimensions, hand shape and some anthropometric characteristics on handgrip strength in male grip athletes and non-atheletes. J. Human Kineti., 29: 151-159.

Islam R, 2018. BBS labour force survey 2016- 17: female labor force growth dwarfs males. The Dhaka Tribune; Accessed 21 April 2019.

Koley S and N Kaur, 2010. An association of nutritional status and handgrip strength in female laborers in north India. The Anthropol., 12: 237-243.

Koley S and Singh AP, 2009. An association of dominant hand grip strength with some anthropometric variables in Indian collegiate population. Anthropol., 67: 21-28.

McGavin G, 2014. The incredible human hand and foot. Retrieved from: http://www.bbc.com/news/scienceenvironment-26224631.

Neves RS, AJ Lopes, SLS Menezes, TRL Lima, AS Ferreira and FS Guimaraes, 2017. Hand grip strength in healthy young and older Brazilian adults: development of a linear prediction model using simple anthropometric variables. Int. J. Kinesiol. Spo. Sci., 49: 208-216.

Oseloka IA, BM Bello, HW Oliver, KU Udua, Emmanuel and MS Abraham, 2014. Association of handgrip strength with body mass index among Nigerian students. J. Pharma. Biol. Sci., 9: 01-07.

Rawat S, LR Varte, IJ Singh, S Choudhary and S Singh, 2016. Anthropometry based prediction of dominant hand grip strength in Indian office going females. Asian J. Medi. Sci., 7: 58- 62.

Ruiz JR, España-Romero V, Ortega FB, Sjöström M, Castillo MJ and Gutierrez A, 2006. Hand span influences optimal grip span in male and female teenagers. J. Hand Surg., 31: 67-72.

Sandhu R, PK Sandhu, D Bajaj and JP Singh, 2017. Anthropometric determinants of hand grip strength in normal healthy Punjabis. Curr. Trends Diag. Treat. 1: 68-71

Shah UN, MS Sirajudeen, PK Somasekaran, N Mohasin and M Shantaram, 2012. The association between hand grip strength and hand dimensions in healthy Indian females. Int. J. Curr. Res. Rev., 4: 36-42.

Vivekanand G, J Rekha and CA Kumar, 2016. Evaluation of hand anthropometric measurements and grip strength in healthy kitchens workers. Int. J. Ana. Physiol. Biochem., 3: 1-5. 